\title{
Erratum: Measurements of the Dynamic Input Impedance of a DC Squid*
}

\author{
Claude Hilbert and John Clarke
}

Department of Physics, University of California, Berkeley, California, and Materials and Molecular Research Division, Lawrence Berkeley Laboratory, Berkeley, California

(Received November 21, 1985)

The curves in Fig. 3 are labeled with incorrect values of $I / I_{0}$. Figure 3 should be replaced by the accompanying figure.

Fig. 3. Dynamic inductance $L / \mathscr{L}$ and dynamic resistance $R / \mathscr{R}$ obtained from the analog simulator for four different values of $I$, as a function of $\Phi$. The SQUID parameters were $\beta=1.0$, $\beta_{c} \approx 0.2$, and $\Gamma \approx 0.06$.

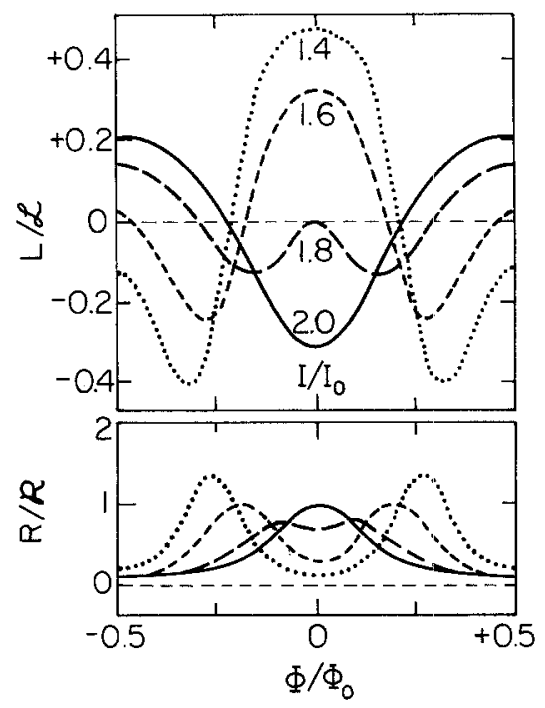

*This paper appeared in J. Low Temp. Phys. 61, 237 (1985). 